\title{
Mecânica Quântica no Espaço de Fase: I. Formulação de Weyl-Wigner
}

(Quantum Mechanics in Phase Space: I. The Weyl-Wigner Formalism)

\author{
Marcelo A. Marchiolli \\ Instituto de Física de São Carlos, Universidade de São Paulo, \\ Caixa Postal 369, 13560-970 São Carlos, SP, Brasil \\ E-mail: marcelo_march@bol.com.br
}

Recebido em 01 de abril, 2002. Aceito em 03 de outubro, 2002.

\begin{abstract}
Nas últimas décadas constata-se um crescente número de artigos em diversas áreas da Física dedicados a desenvolver e aplicar o formalismo de Weyl-Wigner nos mais diferentes sistemas físicos. A possibilidade de aprofundarmos os nossos conhecimentos sobre o princípio da incerteza e recuperarmos a Mecânica Clássica no limite $\hbar \rightarrow 0$, faz com que a descrição da Mecânica Quântica no espaço de fase ganhe cada vez mais adeptos. Nesse sentido, este trabalho tem por finalidade apresentar um conjunto básico de resultados que caracterizam o formalismo em questão, possibilitando ao leitor o acesso às idéias principais decorrentes deste fascinante tema.
\end{abstract}

\begin{abstract}
The last decades have witnessed an ever increasing number of articles in numerous physical areas of research dedicated to develop and apply the Weyl-Wigner formalism in connection with a variety of different physical systems. The possibility of deepening our understanding of the uncertainty principle and recuperating Classical Mechanics in the $\hbar \rightarrow 0$ limit explains the increasing abundance of adepts of the phase-space description of Quantum Mechanics. In this favorable context, the present work aims at introducing a basic set of results that characterize the formalism at issue, allowing the reader to have access to the main ideas emanating from this fascinating theme.
\end{abstract}

\section{Introdução}

O formalismo que iremos desenvolver considera essencialmente um sistema quântico constituído de uma única partícula de massa $M$ executando um movimento unidimensional, nos quais $\mathbf{Q}$ e $\mathbf{P}$ são os respectivos operadores posição e momentum, e destituída de spin (movimento rotacional intrínseco). Como tais operadores satisfazem a relação de comutação $[\mathbf{Q}, \mathbf{P}]=i \hbar \mathbf{1}$, a posição e o momentum desta partícula não podem ser simultaneamente medidos (princípio de incerteza); consequentemente, não é possível definir uma distribuição de probabilidades genuína no espaço de fase associada ao operador densidade $\boldsymbol{\rho}(t)$ que descreve as propriedades físicas da partícula. Nesse sentido, a representação da Mecânica Quântica no espaço de fase é problemática e nada trivial. Entretanto, tais dificuldades foram superadas com o ferramental matemático desenvolvido por Weyl-Wigner [1, 2] em que a função distribuição de Wigner desempenha um papel crucial: esta possui propriedades matemáticas que permitem caracterizá-la como uma pseudo-distribuição (ou quase-distribuição), dentre as quais o fato de poder assumir valores nega- tivos para determinados sistemas quânticos (o que não é permitido no sentido clássico da definição de distribuições de probabilidades). Além disso, a função de Wigner permite estabelecer conexões entre a Mecânica Clássica e a Mecânica Quântica através do limite $\hbar \rightarrow 0$, procedimento esse muito utilizado no estudo de sistemas caóticos semiclássicos $[3,4]$. De fato, as aplicações deste formalismo são inúmeras e se estendem por diferentes áreas da Física [5], sendo que a transição para sistemas tridimensionais e a inclusão do spin podem ser feitas sem mudanças conceituais.

Nos últimos anos, belíssimos experimentos envolvendo a reconstrução de alguns estados do campo eletromagnético [6] ou mesmo de estados associados ao movimento do centro-de-massa de íons ${ }^{9} \mathrm{~B} e{ }^{+}$aprisionados em uma armadilha de Paul [7] foram realizados com sucesso e as respectivas reconstruções feitas por intermédio da função de Wigner. Posteriormente, algumas propostas teóricas para a medida direta da função de Wigner também foram apresentadas [8, 9] e correspondem a experimentos de eletrodinâmica quântica de cavidades e íons aprisionados. Motivados pelos recentes resultados teóricos e experimentais, neste primeiro 
trabalho vamos nos ater somente em realizar um estudo preliminar da formulação de Weyl-Wigner da Mecânica Quântica no espaço de fase, visando a compilação de um material básico que permita ao leitor aprofundarse no tema ou mesmo servir como um complemento às disciplinas dos cursos de pós-graduação em Física, satisfazendo assim os diferentes programas existentes no país.

Os tópicos abordados estão dispostos como segue: na seção 2 deduzimos uma expressão que permite mapear um operador genérico no espaço de fase através de primeiros princípios em Mecânica Quântica (MQ). Esta expressão é interpretada como sendo uma expansão na forma integral de uma base de operadores neste espaço, cujos coeficientes representam a transformada de Weyl associada ao operador genérico em questão. Além disso, obtemos um conjunto razoável de propriedades para os elementos constituintes da expansão e mostramos que a função de Wigner é um caso particular de um formalismo mais geral introduzido por Weyl-Wigner. Na seção 3 calculamos as transformadas de Weyl das relações de comutação e anticomutação para dois operadores também arbitrários, no qual mostramos que tais expressões recuperam resultados já estabelecidos em Mecânica Clássica (MC) no limite $\hbar \rightarrow 0$. Isto nos permite realizar um estudo da evolução temporal da função de Wigner por meio do mapeamento no espaço de fase da equação de von Neumann-Liouville, conforme descrito na seção 4. Tal equação nos leva a obter uma expressão geral para a derivada temporal associada ao valor médio de um observável qualquer que, no caso particular dos operadores momentum e posição, representa a transformada de Weyl das equações de Ehrenfest da formulação usual da MQ. Finalmente, realiza- mos o limite $\hbar \rightarrow 0$ nessas equações com o objetivo de recuperar as equações clássicas de Hamilton da MC. Na seção 5 apresentamos as considerações finais e dicas de leitura para aqueles que queiram se aprofundar no tema. Algumas seções são complementadas com exemplos que visam ilustrar e enriquecer a teoria subjacente.

Este material foi originalmente concebido na forma de apostila e seu conteúdo ministrado no primeiro curso de verão sobre tópicos em Métodos Matemáticos da Fúsica: Introdução ao grupo de rotações tridimensionais, estados coerentes e a formulação de Weyl-Wigner da Mecânica Quântica no espaço de fase, realizado pelo grupo de teoria do Departamento de Física e Ciência dos Materiais (DFCM), do Instituto de Física de São Carlos (IFSC-USP), no período de 21 a 30 de janeiro deste ano e sob a coordenação do Prof. Esmerindo Bernardes. A apostila foi utilizada pelos alunos como material didático e as dúvidas que surgiram ao longo do curso, serviram-me para remodelar o seu conteúdo de modo a transformar os exercícios em exemplos e a incluir novos parágrafos que atendessem as necessidades dos participantes, culminando assim neste trabalho. Por último, gostaria de mencionar que a disposição dos tópicos e algumas passagens matemáticas foram baseadas nas referências $[10,11]$, as quais indico como leitura complementar ao presente texto.

\section{Preliminares Algébricos}

Inicialmente vamos caracterizar a cinemática quântica do sistema físico descrito na introdução, por intermédio das relações de comutação associadas aos operadores $\{\mathbf{Q}, \mathbf{P}, \mathbf{1}\}$, ou seja,

$$
[\mathbf{Q}, \mathbf{Q}]=[\mathbf{P}, \mathbf{P}]=[\mathbf{Q}, \mathbf{1}]=[\mathbf{P}, \mathbf{1}]=0 \quad \text { e } \quad[\mathbf{Q}, \mathbf{P}]=i \hbar \mathbf{1} .
$$

Tais operadores obedecem à álgebra de Weyl-Heisenberg, sendo que os autovetores correspondentes são definidos pelas equações de autovalores

$$
\mathbf{P}|p\rangle=p|p\rangle, \quad \mathbf{Q}|q\rangle=q|q\rangle, \quad \mathbf{1}|p\rangle=|p\rangle, \quad \mathbf{1}|q\rangle=|q\rangle .
$$

As bases dos autovetores $\{|p\rangle\}$ e $\{|q\rangle\}$ satisfazem as relações de completeza e ortonormalização que são dadas, respectivamente, por

$$
\begin{aligned}
& \int_{-\infty}^{\infty} d p|p\rangle\left\langle p\left|=\mathbf{1}, \quad \int_{-\infty}^{\infty} d q\right| q\right\rangle\langle q|=\mathbf{1}, \\
& \left\langle p \mid p^{\prime}\right\rangle=\delta\left(p-p^{\prime}\right), \quad\left\langle q \mid q^{\prime}\right\rangle=\delta\left(q-q^{\prime}\right),
\end{aligned}
$$

sendo $\delta(x)$ a função delta de Dirac. Além disso, também conhecemos o produto escalar

$$
\langle q \mid p\rangle=\frac{1}{\sqrt{2 \pi \hbar}} \exp \left(\frac{i}{\hbar} p q\right),
$$

que permite conectar os diferentes espaços dos autovetores $|p\rangle$ e $|q\rangle$ mediante a transformação de Fourier

$$
|p\rangle=\int_{-\infty}^{\infty} \frac{d q}{\sqrt{2 \pi \hbar}} \exp \left(\frac{i}{\hbar} p q\right)|q\rangle
$$

De posse dessas informações, começaremos então a descrever a formulação de Weyl-Wigner da Mecânica Quântica no espaço de fase.

Considere um operador arbitrário $\mathbf{F}$ tal que seja possível expressá-lo através da identidade 


$$
\begin{aligned}
\mathbf{F} & \equiv \int_{-\infty}^{\infty} d p^{\prime} d p^{\prime \prime} d q^{\prime} d q^{\prime \prime}\left|q^{\prime \prime}\right\rangle\left\langle q^{\prime \prime} \mid p^{\prime \prime}\right\rangle\left\langle p^{\prime \prime}|\mathbf{F}| p^{\prime}\right\rangle\left\langle p^{\prime} \mid q^{\prime}\right\rangle\left\langle q^{\prime}\right| \\
& =\int_{-\infty}^{\infty} \frac{d p^{\prime} d p^{\prime \prime} d q^{\prime} d q^{\prime \prime}}{2 \pi \hbar} \exp \left[\frac{i}{\hbar}\left(p^{\prime \prime} q^{\prime \prime}-p^{\prime} q^{\prime}\right)\right]\left\langle p^{\prime \prime}|\mathbf{F}| p^{\prime}\right\rangle\left|q^{\prime \prime}\right\rangle\left\langle q^{\prime}\right|
\end{aligned}
$$

no qual utilizamos as relações de completeza (3) e o produto escalar (5). Introduzindo as novas variáveis

$$
2 p=p^{\prime}+p^{\prime \prime}, \quad 2 q=q^{\prime}+q^{\prime \prime}, \quad u=p^{\prime \prime}-p^{\prime}, \quad v=q^{\prime \prime}-q^{\prime},
$$

com jacobiano igual a um,

$$
d p^{\prime} d p^{\prime \prime} d q^{\prime} d q^{\prime \prime}=\underbrace{\mathrm{J}\left[\frac{p^{\prime}, p^{\prime \prime}}{p, u}\right]}_{=1} \underbrace{\mathrm{J}\left[\frac{q^{\prime}, q^{\prime \prime}}{q, v}\right]}_{=1} d p d q d u d v=d p d q d u d v
$$

então a identidade (7) pode ser escrita como uma representação integral do operador $\mathbf{F}$, isto é,

$$
\mathbf{F}=\int_{-\infty}^{\infty} \frac{d p d q}{2 \pi \hbar} f(p, q) \Delta(p, q) .
$$

A função $f(p, q)$ é denominada transformada de Weyl do operador $\mathbf{F}$ com relação aos operadores momentum e posição, no qual é dada por $[12,13]$

$$
f(p, q)=\int_{-\infty}^{\infty} d u \exp \left(\frac{i}{\hbar} q u\right)\langle p+u / 2|\mathbf{F}| p-u / 2\rangle .
$$

Note que se $\mathbf{F}$ for um operador hermitiano, então $f(p, q)$ é uma função real. Por sua vez, $\boldsymbol{\Delta}(p, q)$ representa uma base de operadores no espaço de fase e cuja expressão tem a seguinte forma:

$$
\Delta(p, q)=\int_{-\infty}^{\infty} d v \exp \left(\frac{i}{\hbar} p v\right)|q+v / 2\rangle\langle q-v / 2|
$$

A expressão (8) pode ser interpretada como sendo a decomposição do operador $\mathbf{F}$ em uma base de operadores, sendo as componentes $\boldsymbol{\Delta}(p, q)$ os elementos dessa base. Além disso, também mostra que se for conhecida a transformada de Weyl de um dado operador, este pode ser prontamente determinado. Em contrapartida, uma vez conhecido o operador $\mathbf{F}$, a correspondente transformada de Weyl é obtida através da Eq. (9). Existem outras bases estudadas na literatura que apresentam diferentes virtudes para o mapeamento e que podem ser discutidas da mesma maneira como fizemos com a base de Weyl. Um estudo detalhado dessas bases é encontrado na referência [14], no qual é feita uma comparação entre elas.

Exemplo 1 O procedimento matemático realizado para mapear o operador $\mathbf{F}$ não é único. Ao utilizarmos convenientemente as relações de completeza (3) e as demais propriedades estabelecidas no primeiro parágrafo desta seção, obtemos uma representação integral equivalente a Eq. (8), mas agora com expressões alternativas para a transformada de Weyl $f(p, q)$ e a base de operadores $\Delta(p, q)$, isto é,

$$
\begin{aligned}
f(p, q) & =\int_{-\infty}^{\infty} d v \exp \left(\frac{i}{\hbar} p v\right)\langle q-v / 2|\mathbf{F}| q+v / 2\rangle \\
\Delta(p, q) & =\int_{-\infty}^{\infty} d u \exp \left(\frac{i}{\hbar} q u\right)|p-u / 2\rangle\langle p+u / 2|
\end{aligned}
$$

Tais expressões, em conjunto com as Eqs. (9) e (10), permitem demonstrar que os projetores de posição e momentum podem ser escritos, respectivamente, como

$$
\begin{aligned}
|q\rangle\langle q| & =\int_{-\infty}^{\infty} \frac{d u}{2 \pi \hbar} \exp \left[\frac{i}{\hbar} u(q-\mathbf{Q})\right]=\boldsymbol{\delta}(q-\mathbf{Q}) \\
|p\rangle\langle p| & =\int_{-\infty}^{\infty} \frac{d v}{2 \pi \hbar} \exp \left[\frac{i}{\hbar} v(p-\mathbf{P})\right]=\boldsymbol{\delta}(p-\mathbf{P})
\end{aligned}
$$


As integrações nas variáveis u e $v$ nos levam a obter funções delta de Dirac formais, cujo significado está associado com a ação da função delta e a substituição do argumento por um operador, não uma variável. De posse desses resultados e com o auxilio da relação de Baker-Hausdorff ${ }^{1}$, a forma simétrica de $\boldsymbol{\Delta}(p, q)$ é prontamente obtida e dada por

$$
\boldsymbol{\Delta}(p, q)=\int_{-\infty}^{\infty} \frac{d u d v}{2 \pi \hbar} \exp \left\{\frac{i}{\hbar}[u(q-\mathbf{Q})+v(p-\mathbf{P})]\right\}
$$

Em consequência aos resultados delineados até o presente momento, torna-se fácil verificar que a transformada de Weyl admite a forma compacta $f(p, q)=\operatorname{Tr}[\mathbf{F} \boldsymbol{\Delta}(p, q)]$.

Existe um método alternativo muito elegante para se achar o operador $\mathbf{F}$ a partir de sua transformada de Weyl. Para tanto, considere inicialmente a expressão do operador $\boldsymbol{\Delta}(p, q)$ escrita como

$$
\boldsymbol{\Delta}(p, q)=\int_{-\infty}^{\infty} \frac{d u d v}{2 \pi \hbar} \exp \left[\frac{i}{\hbar} u(q-\mathbf{Q})\right] \exp \left[\frac{i}{\hbar} v(p-\mathbf{P})\right] \exp \left(\frac{i}{\hbar} \frac{u v}{2}\right) .
$$

Em seguida, verifique que a igualdade

$$
\frac{\hbar}{2 i} \frac{\partial^{2}}{\partial p \partial q} \exp \left[\frac{i}{\hbar} u(q-\mathbf{Q})\right] \exp \left[\frac{i}{\hbar} v(p-\mathbf{P})\right]=\frac{i}{\hbar} \frac{u v}{2} \exp \left[\frac{i}{\hbar} u(q-\mathbf{Q})\right] \exp \left[\frac{i}{\hbar} v(p-\mathbf{P})\right]
$$

nos leva a obter o integrando da Eq. (16) através da operação

$$
\begin{aligned}
\sum_{k=0}^{\infty} \frac{1}{k !}\left(\frac{\hbar}{2 i} \frac{\partial^{2}}{\partial p \partial q}\right)^{k} \exp \left[\frac{i}{\hbar} u(q-\mathbf{Q})\right] \exp \left[\frac{i}{\hbar} v(p-\mathbf{P})\right]= & \sum_{k=0}^{\infty} \frac{1}{k !}\left(\frac{i}{\hbar} \frac{u v}{2}\right)^{k} \exp \left[\frac{i}{\hbar} u(q-\mathbf{Q})\right] \\
& \times \exp \left[\frac{i}{\hbar} v(p-\mathbf{P})\right],
\end{aligned}
$$

ou seja,

$$
\begin{aligned}
\exp \left(\frac{\hbar}{2 i} \frac{\partial^{2}}{\partial p \partial q}\right) \exp \left[\frac{i}{\hbar} u(q-\mathbf{Q})\right] \exp \left[\frac{i}{\hbar} v(p-\mathbf{P})\right]= & \exp \left(\frac{i}{\hbar} \frac{u v}{2}\right) \exp \left[\frac{i}{\hbar} u(q-\mathbf{Q})\right] \\
& \times \exp \left[\frac{i}{\hbar} v(p-\mathbf{P})\right]
\end{aligned}
$$

Agora, inserindo esse resultado na expressão para $\boldsymbol{\Delta}(p, q)$ chega-se a

$$
\begin{aligned}
\boldsymbol{\Delta}(p, q) & =\exp \left(\frac{\hbar}{2 i} \frac{\partial^{2}}{\partial p \partial q}\right) \int_{-\infty}^{\infty} \frac{d u d v}{2 \pi \hbar} \exp \left[\frac{i}{\hbar} u(q-\mathbf{Q})\right] \exp \left[\frac{i}{\hbar} v(p-\mathbf{P})\right] \\
& =2 \pi \hbar \exp \left(\frac{\hbar}{2 i} \frac{\partial^{2}}{\partial p \partial q}\right) \boldsymbol{\delta}(q-\mathbf{Q}) \boldsymbol{\delta}(p-\mathbf{P})
\end{aligned}
$$

o qual permite-nos escrever a expressão para o operador $\mathbf{F}$ como

$$
\begin{aligned}
\mathbf{F} & =\int_{-\infty}^{\infty} d p d q f(p, q)\left[\exp \left(\frac{\hbar}{2 i} \frac{\partial^{2}}{\partial p \partial q}\right) \boldsymbol{\delta}(q-\mathbf{Q}) \boldsymbol{\delta}(p-\mathbf{P})\right] \\
& =\int_{-\infty}^{\infty} d p d q\left[\exp \left(\frac{\hbar}{2 i} \frac{\partial^{2}}{\partial p \partial q}\right) f(p, q)\right] \boldsymbol{\delta}(q-\mathbf{Q}) \boldsymbol{\delta}(p-\mathbf{P})
\end{aligned}
$$

sendo a segunda igualdade obtida por intermédio da relação auxiliar

$$
\int_{-\infty}^{\infty} d x f(x)\left[\frac{d^{m}}{d x^{m}} \delta(x)\right]=\left.(-1)^{m} \frac{d^{m}}{d x^{m}} f(x)\right|_{x=0}=(-1)^{m} \int_{-\infty}^{\infty} d x\left[\frac{d^{m}}{d x^{m}} f(x)\right] \delta(x) .
$$

\footnotetext{
${ }^{1}$ Se $\mathbf{A}$ e $\mathbf{B}$ são dois operadores que não comutam e que satisfazem as condições

$$
[\mathbf{A},[\mathbf{A}, \mathbf{B}]]=[\mathbf{B},[\mathbf{A}, \mathbf{B}]]=0,
$$

então

$$
e^{\mathbf{A}+\mathbf{B}}=e^{\mathbf{A}} e^{\mathbf{B}} e^{-\frac{1}{2}[\mathbf{A}, \mathbf{B}]}=e^{\mathbf{B}} e^{\mathbf{A}} e^{\frac{1}{2}[\mathbf{A}, \mathbf{B}]} .
$$

Este resultado é uma forma simplificada da relação de Baker-Hausdorff [15].
} 
Note que para encontrarmos tal operador, a partir de sua transformada de Weyl, devemos em um primeiro momento calcular

$$
\exp \left(\frac{\hbar}{2 i} \frac{\partial^{2}}{\partial p \partial q}\right) f(p, q)
$$

e depois substituir as variáveis $q$ e $p$ pelos respectivos operadores $\mathbf{Q}$ e $\mathbf{P}$, com a ressalva de escrevermos os operadores posição à esquerda dos operadores momentum. Este procedimento é muito útil quando se trabalha com produtos simples de $\mathbf{Q}$ e $\mathbf{P}$, e a ressalva da ordem dos operadores garante a completa simetrização do operador resultante [10].

Exemplo 2 Como aplicação do método estabelecido no parágrafo anterior, considere que a transformada de Weyl seja conhecida e dada pela função $f(p, q)=g(q) p^{n}$. Assim sendo, temos que

$$
\begin{aligned}
\exp \left(\frac{\hbar}{2 i} \frac{\partial^{2}}{\partial p \partial q}\right) g(q) p^{n} & =\sum_{k=0}^{\infty} \frac{1}{k !}\left(\frac{\hbar}{2 i}\right)^{k} \frac{\partial^{k}}{\partial q^{k}} g(q) \frac{\partial^{k}}{\partial p^{k}} p^{n} \\
& =\sum_{k=0}^{n}\left(\begin{array}{c}
n \\
k
\end{array}\right)\left(\frac{\hbar}{2 i}\right)^{k} \frac{\partial^{k}}{\partial q^{k}} g(q) p^{n-k}
\end{aligned}
$$

no qual $\left(\begin{array}{c}n \\ k\end{array}\right) \equiv n ! / k !(n-k)$ ! denota os coeficientes binomiais. A segunda igualdade foi obtida com o auxilio da relação

$$
\frac{\partial^{k}}{\partial p^{k}} p^{n}=\frac{n !}{(n-k) !} p^{n-k} \quad(k \leq n),
$$

levando-nos a transformar a soma infinita em uma soma finita. Ao substituirmos esse resultado na Eq. (18) e realizando as integrações com o cuidado de preservar a ordem dos operadores posição e momentum, chega-se a

$$
\mathbf{F}=\sum_{k=0}^{n}\left(\begin{array}{c}
n \\
k
\end{array}\right)\left(\frac{\hbar}{2 i}\right)^{k} \frac{d^{k}}{d \mathbf{Q}^{k}} \mathbf{g}(\mathbf{Q}) \mathbf{P}^{n-k}
$$

Em seguida, vamos demonstrar que o operador $\mathbf{F}$ também pode ser escrito como

$$
\mathbf{F}=\frac{1}{2^{n}} \sum_{l=0}^{n}\left(\begin{array}{c}
n \\
l
\end{array}\right) \mathbf{P}^{l} \mathbf{g}(\mathbf{Q}) \mathbf{P}^{n-l}
$$

Para tanto, recorreremos inicialmente as relações já estabelecidas na literatura para os coeficientes binomiais,

$$
\left(\begin{array}{c}
n \\
l
\end{array}\right)\left(\begin{array}{l}
l \\
k
\end{array}\right)=\left(\begin{array}{c}
n \\
k
\end{array}\right)\left(\begin{array}{c}
n-k \\
l-k
\end{array}\right) \quad \text { e } \quad \sum_{m=0}^{n-k}\left(\begin{array}{c}
n-k \\
m
\end{array}\right)=2^{n-k}
$$

de modo a obtermos uma expressão intermediária para o operador $\mathbf{F}$ mediante os seguinte passos:

$$
\begin{aligned}
\mathbf{F} & =\frac{1}{2^{n}} \sum_{k=0}^{n}\left(\begin{array}{c}
n \\
k
\end{array}\right) 2^{n-k}\left(\frac{\hbar}{i}\right)^{k} \frac{\partial^{k}}{\partial \mathbf{Q}^{k}} \mathbf{g}(\mathbf{Q}) \mathbf{P}^{n-k} \\
& =\frac{1}{2^{n}} \sum_{k=0}^{n} \sum_{m=0}^{n-k}\left(\begin{array}{c}
n \\
k
\end{array}\right)\left(\begin{array}{c}
n-k \\
m
\end{array}\right)\left(\frac{\hbar}{i}\right)^{k} \frac{\partial^{k}}{\partial \mathbf{Q}^{k}} \mathbf{g}(\mathbf{Q}) \mathbf{P}^{n-k}
\end{aligned}
$$

Agora, realizando a troca de índices $m=l-k$ com $k \leq l \leq n$ na segunda soma, obtem-se

$$
\begin{aligned}
\mathbf{F} & =\frac{1}{2^{n}} \sum_{k=0}^{n} \sum_{l=k}^{n}\left(\begin{array}{c}
n \\
k
\end{array}\right)\left(\begin{array}{c}
n-k \\
l-k
\end{array}\right)\left(\frac{\hbar}{i}\right)^{k} \frac{\partial^{k}}{\partial \mathbf{Q}^{k}} \mathbf{g}(\mathbf{Q}) \mathbf{P}^{n-k} \\
& =\frac{1}{2^{n}} \sum_{l=0}^{n} \sum_{k=0}^{l}\left(\begin{array}{c}
n \\
l
\end{array}\right)\left(\begin{array}{c}
l \\
k
\end{array}\right)\left(\frac{\hbar}{i}\right)^{k} \frac{\partial^{k}}{\partial \mathbf{Q}^{k}} \mathbf{g}(\mathbf{Q}) \mathbf{P}^{n-k} \\
& =\frac{1}{2^{n}} \sum_{l=0}^{n}\left(\begin{array}{c}
n \\
l
\end{array}\right) \underbrace{\sum_{k=0}^{l}\left(\begin{array}{c}
l \\
k
\end{array}\right)\left(\frac{\hbar}{i}\right)^{k} \frac{\partial^{k}}{\partial \mathbf{Q}^{k}} \mathbf{g}(\mathbf{Q}) \mathbf{P}^{l-k}}_{\mathbf{P}^{l} \mathbf{g}(\mathbf{Q})} \mathbf{P}^{n-l} \\
& =\frac{1}{2^{n}} \sum_{l=0}^{n}\left(\begin{array}{c}
n \\
l
\end{array}\right) \mathbf{P}^{l} \mathbf{g}(\mathbf{Q}) \mathbf{P}^{n-l} .
\end{aligned}
$$


Note que na terceira igualdade utilizamos a relação de comutação $[\mathbf{P}, \mathbf{g}(\mathbf{Q})]=-i \hbar \frac{d}{d \mathbf{Q}} \mathbf{g}(\mathbf{Q})$, o que permitiu-nos mostrar a relação

$$
\mathbf{P}^{l} \mathbf{g}(\mathbf{Q})=\sum_{k=0}^{l}\left(\begin{array}{c}
l \\
k
\end{array}\right)\left(\frac{\hbar}{i}\right)^{k} \frac{d^{k}}{d \mathbf{Q}^{k}} \mathbf{g}(\mathbf{Q}) \mathbf{P}^{l-k}
$$

Por último, resta-nos somente mencionar o fato de que a Eq. (20) possui uma expressão analítica fechada que é dada por

$$
\mathbf{F}=\frac{1}{2^{n}} \underbrace{\{\{\cdots\{\mathbf{g}(\mathbf{Q}), \mathbf{P}\}, \mathbf{P}\}, \cdots, \mathbf{P}\}}_{n \text { anticomutadores }},
$$

em que $\{\mathbf{A}, \mathbf{B}\}=\mathbf{A B}+\mathbf{B A}$ corresponde ao anticomutador entre os operadores $\mathbf{A}$ e $\mathbf{B}$. Para deduzirmos tal expressão, basta recorrermos a relação de comutação supracitada para verificarmos que

$$
\underbrace{\{\{\cdots\{\mathbf{g}(\mathbf{Q}), \mathbf{P}\}, \mathbf{P}\}, \cdots, \mathbf{P}\}}_{n \text { anticomutadores }}=\sum_{l=0}^{n}\left(\begin{array}{c}
n \\
l
\end{array}\right) \mathbf{P}^{l} \mathbf{g}(\mathbf{Q}) \mathbf{P}^{n-l},
$$

levando-nos assim ao resultado desejado.

Já a função da Mecânica Clássica que corresponde ao operador $\mathbf{F}$, pode ser encontrada por intermédio da transformada de Weyl deste operador realizando-se o limite $\hbar \rightarrow 0$,

$$
f_{c l}(p, q)=\lim _{\hbar \rightarrow 0} f(p, q)
$$

Note que $f(p, q)$ não é a quantidade clássica associada ao operador quântico $\mathbf{F}$, mas somente uma função que, em geral, pode ser expressa como uma série de potências em $\hbar$. Portanto, o procedimento $\hbar \rightarrow 0$ é crucial para obtermos $f_{c l}(p, q)$.

No formalismo desenvolvido até o presente mo- mento, $\boldsymbol{\Delta}(p, q)$ desempenha um papel importante pois trata-se de um elemento que permite mapear operadores no espaço de fase. Como tal, este também pode ser expandido numa base de operadores como

$$
\boldsymbol{\Delta}\left(p^{\prime}, q^{\prime}\right)=\int_{-\infty}^{\infty} \frac{d p d q}{2 \pi \hbar} f(p, q) \Delta(p, q)
$$

no qual $f(p, q)=2 \pi \hbar \delta\left(p-p^{\prime}\right) \delta\left(q-q^{\prime}\right)$. Em seguida, vamos exibir uma série de propriedades para $\boldsymbol{\Delta}(p, q)$ que serão muito úteis no decorrer do trabalho. Dessa forma, vamos iniciar com os elementos de matriz

$$
\begin{aligned}
\text { (i) }\left\langle q^{\prime}|\boldsymbol{\Delta}(p, q)| q^{\prime \prime}\right\rangle & =\exp \left[\frac{i}{\hbar} p\left(q^{\prime}-q^{\prime \prime}\right)\right] \delta\left(q-\frac{q^{\prime}+q^{\prime \prime}}{2}\right), \\
\text { (ii) }\left\langle p^{\prime}|\boldsymbol{\Delta}(p, q)| p^{\prime \prime}\right\rangle & =\exp \left[-\frac{i}{\hbar} q\left(p^{\prime}-p^{\prime \prime}\right] \delta\left(p-\frac{p^{\prime}+p^{\prime \prime}}{2}\right),\right. \\
\text { (iii) }\left\langle q^{\prime}|\boldsymbol{\Delta}(p, q)| p^{\prime}\right\rangle & =\sqrt{2 \pi \hbar} \exp \left(\frac{i}{\hbar} p^{\prime} q^{\prime}\right) \exp \left(\frac{\hbar}{2 i} \frac{\partial^{2}}{\partial p \partial q}\right) \delta\left(p-p^{\prime}\right) \delta\left(q-q^{\prime}\right) .
\end{aligned}
$$

As Eqs.(23) e (24) podem ser demonstradas de imediato com o auxílio das definições estabelecidas para $\boldsymbol{\Delta}(p, q)$ e o produto escalar (5). Consequentemente, ao considerarmos $q^{\prime \prime}=q^{\prime}$ e $p^{\prime \prime}=p^{\prime}$, tais expressões se reduzem a $\left\langle q^{\prime}|\boldsymbol{\Delta}(p, q)| q^{\prime}\right\rangle=\delta\left(q-q^{\prime}\right)$ e $\left\langle p^{\prime}|\boldsymbol{\Delta}(p, q)| p^{\prime}\right\rangle=\delta\left(p-p^{\prime}\right)$, respectivamente. Com relação a demonstração da Eq.(25), esta requer o auxílio da Eq.(17) e alguns cuidados decorrentes do cálculo como veremos abaixo:

$$
\begin{aligned}
\left\langle q^{\prime}|\boldsymbol{\Delta}(p, q)| p^{\prime}\right\rangle & =2 \pi \hbar \exp \left(\frac{\hbar}{2 i} \frac{\partial^{2}}{\partial p \partial q}\right)\left\langle q^{\prime}|\boldsymbol{\delta}(q-\mathbf{Q}) \boldsymbol{\delta}(p-\mathbf{P})| p^{\prime}\right\rangle \\
& =2 \pi \hbar \exp \left(\frac{\hbar}{2 i} \frac{\partial^{2}}{\partial p \partial q}\right) \int_{-\infty}^{\infty} d q^{\prime \prime}\left\langle q^{\prime}|\boldsymbol{\delta}(q-\mathbf{Q})| q^{\prime \prime}\right\rangle\left\langle q^{\prime \prime}|\boldsymbol{\delta}(p-\mathbf{P})| p^{\prime}\right\rangle \\
& =2 \pi \hbar \exp \left(\frac{\hbar}{2 i} \frac{\partial^{2}}{\partial p \partial q}\right) \delta\left(p-p^{\prime}\right) \int_{-\infty}^{\infty} \frac{d q^{\prime \prime}}{\sqrt{2 \pi \hbar}} \exp \left(\frac{i}{\hbar} p^{\prime} q^{\prime \prime}\right) \delta\left(q-q^{\prime \prime}\right) \delta\left(q^{\prime}-q^{\prime \prime}\right) \\
& =\sqrt{2 \pi \hbar} \exp \left(\frac{i}{\hbar} p^{\prime} q^{\prime}\right) \exp \left(\frac{\hbar}{2 i} \frac{\partial^{2}}{\partial p \partial q}\right) \delta\left(p-p^{\prime}\right) \delta\left(q-q^{\prime}\right) .
\end{aligned}
$$


As integrações do operador $\boldsymbol{\Delta}(p, q)$ nas variáveis $p$ e $q$ resultam em

$$
\text { (iv) } \int_{-\infty}^{\infty} \frac{d p}{2 \pi \hbar} \Delta(p, q)=|q\rangle\langle q|
$$

e

$$
\text { (v) } \int_{-\infty}^{\infty} \frac{d q}{2 \pi \hbar} \boldsymbol{\Delta}(p, q)=|p\rangle\langle p| .
$$

Tais resultados nos levam aos projetores dos estados particulares do conjunto de autovetores dos operadores posição e momentum. Como decorrência dessas propriedades, vamos retornar à definição da transformada de Weyl do operador $\mathbf{F}$, ou seja, $f(p, q)=\operatorname{Tr}[\mathbf{F} \boldsymbol{\Delta}(p, q)]$. Ao considerarmos $\mathbf{F}=\boldsymbol{\rho}$ (operador densidade associado a um sistema físico), obtemos formalmente a definição da função de Wigner [2]

$$
W(p, q)=\int_{-\infty}^{\infty} d v \exp \left(\frac{i}{\hbar} p v\right)\langle q-v / 2|\rho| q+v / 2\rangle
$$

Assim, para $\rho=|\Psi\rangle\langle\Psi|$, as propriedades (26) e (27) assumem a seguinte forma:

$$
\int_{-\infty}^{\infty} \frac{d p}{2 \pi \hbar} W(p, q)=|\Psi(q)|^{2} \quad \text { e } \quad \int_{-\infty}^{\infty} \frac{d q}{2 \pi \hbar} W(p, q)=|\Phi(p)|^{2} .
$$

Logo, ao integrarmos a função de Wigner em uma das variáveis do espaço de fase, recuperamos as densidades de probabilidades associadas às respectivas funções de onda $\Psi(q)$ e $\Phi(p)$. Esta é uma característica particular de $W(p, q)$ e não se estende para as demais quase-distribuições de probabilidades [16, 17]. Outras propriedades serão abordadas nas próximas seções.

Exemplo 3 Vamos calcular a função de Wigner associada ao n-ésimo estado do oscilador harmônico unidimensional descrito pelo operador densidade $\boldsymbol{\rho}=|n\rangle\langle n|$ e cuja função de onda é dada por

$$
\Psi_{n}(q)=\langle q \mid n\rangle=\frac{1}{\sqrt{\sqrt{\pi} 2^{n} n ! b}} \exp \left(-\frac{q^{2}}{2 b^{2}}\right) H_{n}(q / b),
$$

em que $H_{n}(x)$ denota os polinômios de Hermite e $b^{2}=\hbar / m \omega$. Para tanto, devemos inicialmente recorrer a Eq. (28) e proceder com o cálculo da integral delineado abaixo:

$$
W_{n}(p, q)=\frac{2(-1)^{n}}{\sqrt{\pi} 2^{n} n !} \exp \left[-\left(\frac{q^{2}}{b^{2}}+\frac{b^{2} p^{2}}{\hbar^{2}}\right)\right] \int_{-\infty}^{\infty} \frac{d v}{2 b} \exp \left[-\left(\frac{v}{2 b}-i \frac{b p}{\hbar}\right)^{2}\right] H_{n}\left(\frac{v}{2 b}-\frac{q}{b}\right) H_{n}\left(\frac{v}{2 b}+\frac{q}{b}\right)
$$

Substituindo a variável $x=\frac{v}{2 b}-i \frac{b p}{\hbar}$ no integrando, obtem-se a expressão intermediária

$$
W_{n}(p, q)=\frac{2(-1)^{n}}{\sqrt{\pi} 2^{n} n !} \exp \left[-\left(\frac{q^{2}}{b^{2}}+\frac{b^{2} p^{2}}{\hbar^{2}}\right)\right] \int_{-\infty}^{\infty} d x e^{-x^{2}} H_{n}\left[x+\left(\frac{q}{b}+i \frac{b p}{\hbar}\right)\right] H_{n}\left[x-\left(\frac{q}{b}-i \frac{b p}{\hbar}\right)\right] .
$$

Agora, com o auxilio da relação [18]

$$
\int_{-\infty}^{\infty} d x e^{-x^{2}} H_{m}(x+y) H_{n}(x+z)=\sqrt{\pi} 2^{n} m ! z^{n-m} L_{m}^{(n-m)}(-2 y z) \quad(n \geq m)
$$

sendo $L_{n}^{(m)}(x)$ os polinômios de Laguerre associados, a integração pode ser efetuada de imediato, levando-nos ao resultado final

$$
W_{n}(p, q)=2(-1)^{n} \exp \left[-\left(\frac{q^{2}}{b^{2}}+\frac{b^{2} p^{2}}{\hbar^{2}}\right)\right] L_{n}\left[2\left(\frac{q^{2}}{b^{2}}+\frac{b^{2} p^{2}}{\hbar^{2}}\right)\right] .
$$

Esta função é comumente encontrada em livros textos sobre Óptica Quântica [19-22] sob a forma compacta

$$
W_{n}(\alpha)=2(-1)^{n} e^{-|\alpha|^{2}} L_{n}\left(2|\alpha|^{2}\right)
$$

com $\alpha=\frac{q}{b}+i \frac{b p}{\hbar}$. A figura 1 mostra os gráficos da função de Wigner $W_{n}(p, q)$ versus $\left(\frac{q}{b}, \frac{b p}{\hbar}\right)$ para o estado fundamental (a) $n=0$ e os cinco primeiros estados excitados (b)-(f) $n=1, \ldots, 5$ do oscilador harmônico unidimensional. Note que a função assume valores negativos no espaço de fase e este importante fato está associado ao caráter quântico do operador densidade em questão. É importante salientarmos que a função $W_{1}(p, q)$ foi reconstruída recentemente pelo grupo do NIST em um belo experimento envolvendo ions ${ }^{9}$ Be ${ }^{+}$aprisionados em uma armadilha de Paul [7]. Na seção IV, retomaremos novamente neste ponto ao discutirmos as propriedades da função de Wigner. 
(a) $\mathrm{n}=0$

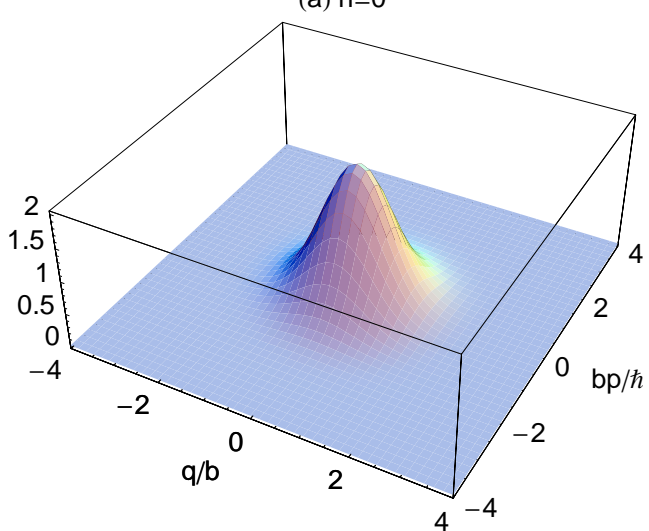

(b) $n=1$

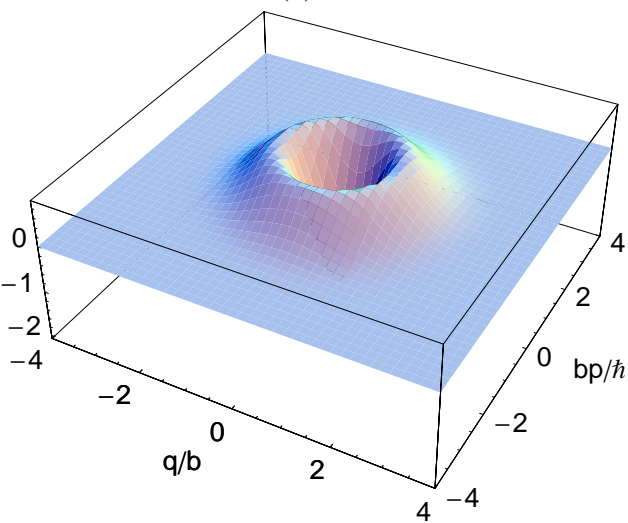

(c) $\mathrm{n}=2$

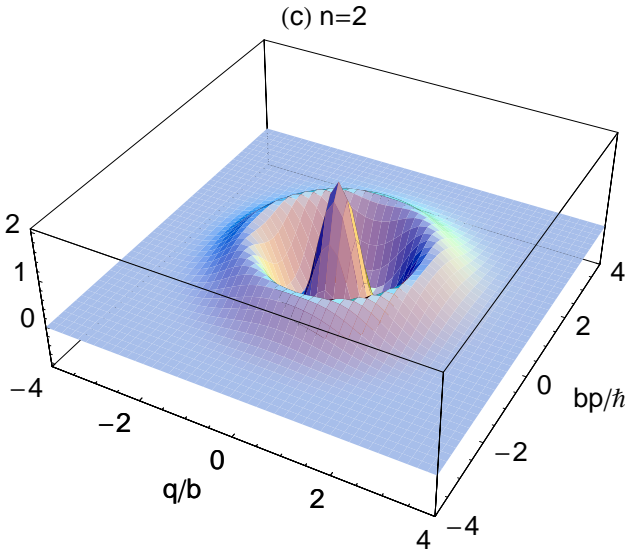

(d) $n=3$

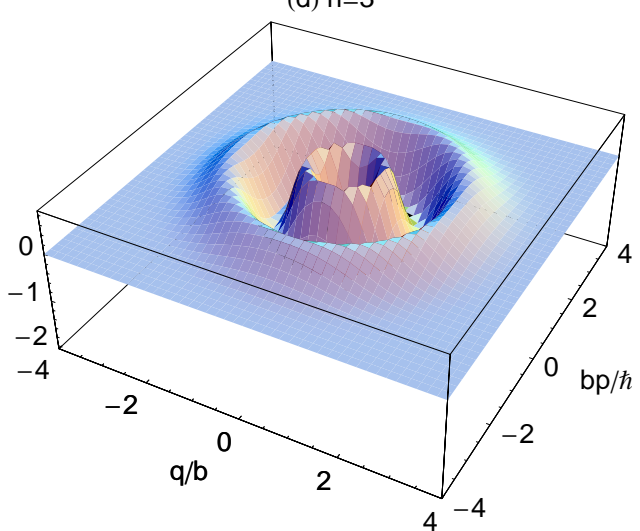

(e) $n=4$

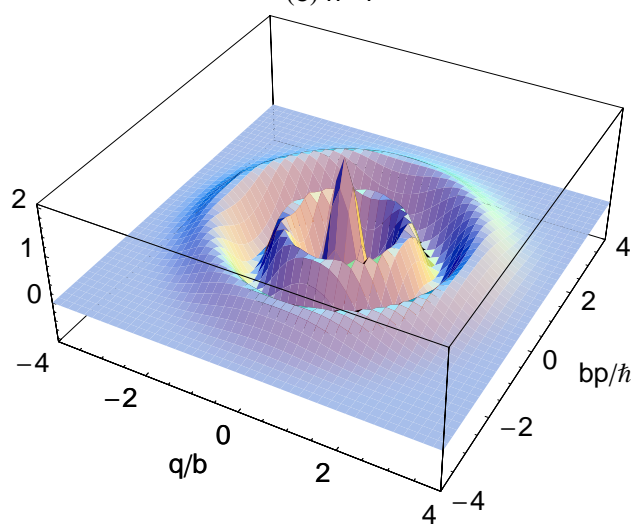

(f) $n=5$

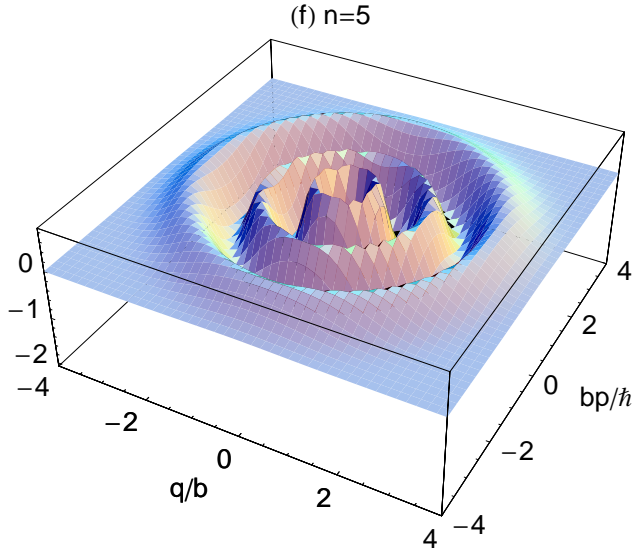

Figura 1. As figuras 1(a)-(f) correspondem aos gráficos de $W_{n}(p, q)$ versus $\frac{q}{b}$ e $\frac{b p}{\hbar}$ para $n=0, \ldots, 5$. Os valores negativos da função de Wigner estão compreendidos no intervalo $-\frac{1}{\pi \hbar} \leq W_{n}(p, q) \leq \frac{1}{\pi \hbar}$ e associados ao caráter quântico do estado de número.

Para finalizarmos esta seção, resta-nos somente mencionar as propriedades relativas ao traço do operador $\boldsymbol{\Delta}(p, q)$ e do produto destes operadores, com a finalidade de obtermos expressões analíticas para a transformada de Weyl das relações de comutação e anticomutação. Recorrendo a definição da operação traço, temos que

(vi) $\operatorname{Tr}[\boldsymbol{\Delta}(p, q)]=1$,

(vii) $\operatorname{Tr}\left[\boldsymbol{\Delta}\left(p_{1}, q_{1}\right) \boldsymbol{\Delta}\left(p_{2}, q_{2}\right)\right]=2 \pi \hbar \delta\left(p_{1}-p_{2}\right) \delta\left(q_{1}-q_{2}\right)$,

(viii) $\operatorname{Tr}\left[\boldsymbol{\Delta}\left(p_{1}, q_{1}\right) \boldsymbol{\Delta}\left(p_{2}, q_{2}\right) \boldsymbol{\Delta}\left(p_{3}, q_{3}\right)\right]=2^{2} \exp \left\{\frac{2 i}{\hbar}\left[\left(q_{1}-q_{3}\right)\left(p_{2}-p_{3}\right)-\left(q_{2}-q_{3}\right)\left(p_{1}-p_{3}\right)\right]\right\}$. 
A demonstração da Eq.(34) é imediata desde que utilizamos a propriedade (23), ou seja,

$$
\begin{aligned}
\operatorname{Tr}\left[\boldsymbol{\Delta}\left(p_{1}, q_{1}\right) \boldsymbol{\Delta}\left(p_{2}, q_{2}\right)\right] & =\int_{-\infty}^{\infty} d q^{\prime}\left\langle q^{\prime}\left|\boldsymbol{\Delta}\left(p_{1}, q_{1}\right) \boldsymbol{\Delta}\left(p_{2}, q_{2}\right)\right| q^{\prime}\right\rangle \\
& =\int_{-\infty}^{\infty} d q^{\prime} d q^{\prime \prime}\left\langle q^{\prime}\left|\boldsymbol{\Delta}\left(p_{1}, q_{1}\right)\right| q^{\prime \prime}\right\rangle\left\langle q^{\prime \prime}\left|\boldsymbol{\Delta}\left(p_{2}, q_{2}\right)\right| q^{\prime}\right\rangle \\
& =\int_{-\infty}^{\infty} d q^{\prime} d q^{\prime \prime} \exp \left[\frac{i}{\hbar}\left(q^{\prime}-q^{\prime \prime}\right)\left(p_{1}-p_{2}\right)\right] \delta\left(q_{1}-\frac{q^{\prime}+q^{\prime \prime}}{2}\right) \delta\left(q_{2}-\frac{q^{\prime}+q^{\prime \prime}}{2}\right) .
\end{aligned}
$$

Agora, substituindo as novas variáveis $q^{\prime}+q^{\prime \prime}=2 x_{1}$ e $q^{\prime}-q^{\prime \prime}=x_{2}$ na expressão acima com

$$
d q^{\prime} d q^{\prime \prime}=\underbrace{\mathrm{J}\left[\frac{q^{\prime}, q^{\prime \prime}}{x_{1}, x_{2}}\right]}_{=1} d x_{1} d x_{2}=d x_{1} d x_{2}
$$

finalizamos os cálculos como segue:

$$
\begin{aligned}
\operatorname{Tr}\left[\boldsymbol{\Delta}\left(p_{1}, q_{1}\right) \boldsymbol{\Delta}\left(p_{2}, q_{2}\right)\right] & =\int_{-\infty}^{\infty} d x_{1} d x_{2} \exp \left[\frac{i}{\hbar} x_{2}\left(p_{1}-p_{2}\right)\right] \delta\left(q_{1}-x_{1}\right) \delta\left(q_{2}-x_{2}\right) \\
& =2 \pi \hbar \delta\left(p_{1}-p_{2}\right) \delta\left(q_{1}-q_{2}\right) .
\end{aligned}
$$

De maneira análoga, temos que a demonstração da Eq.(35) exige cuidados adicionais, uma vez que

$$
\begin{aligned}
\operatorname{Tr}\left[\boldsymbol{\Delta}\left(p_{1}, q_{1}\right) \boldsymbol{\Delta}\left(p_{2}, q_{2}\right) \boldsymbol{\Delta}\left(p_{3}, q_{3}\right)\right]= & \int_{-\infty}^{\infty} d q^{\prime} d q^{\prime \prime} d q^{\prime \prime \prime}\left\langle q^{\prime}\left|\boldsymbol{\Delta}\left(p_{1}, q_{1}\right)\right| q^{\prime \prime}\right\rangle\left\langle q^{\prime \prime}\left|\boldsymbol{\Delta}\left(p_{2}, q_{2}\right)\right| q^{\prime \prime \prime}\right\rangle\left\langle q^{\prime \prime \prime}\left|\boldsymbol{\Delta}\left(p_{3}, q_{3}\right)\right| q^{\prime}\right\rangle \\
= & \int_{-\infty}^{\infty} d q^{\prime} d q^{\prime \prime} d q^{\prime \prime \prime} \exp \left\{\frac{i}{\hbar}\left[p_{1}\left(q^{\prime}-q^{\prime \prime}\right)+p_{2}\left(q^{\prime \prime}-q^{\prime \prime \prime}\right)+p_{3}\left(q^{\prime \prime \prime}-q^{\prime}\right)\right]\right\} \\
& \times \delta\left(q_{1}-\frac{q^{\prime}+q^{\prime \prime}}{2}\right) \delta\left(q_{2}-\frac{q^{\prime \prime}+q^{\prime \prime \prime}}{2}\right) \delta\left(q_{3}-\frac{q^{\prime \prime \prime}+q^{\prime}}{2}\right) .
\end{aligned}
$$

Agora, designando $q^{\prime}+q^{\prime \prime}=2 x_{1}, q^{\prime \prime}+q^{\prime \prime \prime}=2 x_{2}$ e $q^{\prime \prime \prime}+q^{\prime}=2 x_{3}$ com

$$
d q^{\prime} d q^{\prime \prime} d q^{\prime \prime \prime}=\underbrace{\mathrm{J}\left[\frac{q^{\prime}, q^{\prime \prime}, q^{\prime \prime \prime}}{x_{1}, x_{2}, x_{3}}\right]}_{=2^{2}} d x_{1} d x_{2} d x_{3}=2^{2} d x_{1} d x_{2} d x_{3},
$$

obtemos

$$
\begin{aligned}
\operatorname{Tr}\left[\boldsymbol{\Delta}\left(p_{1}, q_{1}\right) \boldsymbol{\Delta}\left(p_{2}, q_{2}\right) \boldsymbol{\Delta}\left(p_{3}, q_{3}\right)\right]= & 2^{2} \int_{-\infty}^{\infty} d x_{1} d x_{2} d x_{3} \delta\left(q_{1}-x_{1}\right) \delta\left(q_{2}-x_{2}\right) \delta\left(q_{3}-x_{3}\right) \\
& \times \exp \left\{\frac{2 i}{\hbar}\left[\left(x_{1}-x_{3}\right)\left(p_{2}-p_{3}\right)-\left(x_{2}-x_{3}\right)\left(p_{1}-p_{3}\right)\right]\right\} \\
= & 2^{2} \exp \left\{\frac{2 i}{\hbar}\left[\left(q_{1}-q_{3}\right)\left(p_{2}-p_{3}\right)-\left(q_{2}-q_{3}\right)\left(p_{1}-p_{3}\right)\right]\right\} .
\end{aligned}
$$

Consequentemente, ao identificarmos novamente $\mathbf{F}$ com o operador densidade $\boldsymbol{\rho}$, novas propriedades associadas a função de Wigner surgem naturalmente: a primeira refere-se a sua normalização,

$$
\int_{-\infty}^{\infty} \frac{d p d q}{2 \pi \hbar} W(p, q)=1
$$

enquanto que a segunda propriedade reflete a ciclicidade da operação traço,

$$
\begin{aligned}
\operatorname{Tr}\left(\boldsymbol{\rho}_{1} \boldsymbol{\rho}_{2}\right) & =\int_{-\infty}^{\infty} \frac{d p_{1} d q_{1} d p_{2} d q_{2}}{(2 \pi \hbar)^{2}} W_{\boldsymbol{\rho}_{1}}\left(p_{1}, q_{1}\right) W_{\boldsymbol{\rho}_{2}}\left(p_{2}, q_{2}\right) \operatorname{Tr}\left[\boldsymbol{\Delta}\left(p_{1}, q_{1}\right) \boldsymbol{\Delta}\left(p_{2}, q_{2}\right)\right] \\
& =\int_{-\infty}^{\infty} \frac{d p_{1} d q_{1} d p_{2} d q_{2}}{2 \pi \hbar} W_{\boldsymbol{\rho}_{1}}\left(p_{1}, q_{1}\right) W \boldsymbol{\rho}_{2}\left(p_{2}, q_{2}\right) \delta\left(p_{1}-p_{2}\right) \delta\left(q_{1}-q_{2}\right) \\
& =\int_{-\infty}^{\infty} \frac{d p_{1} d q_{1}}{2 \pi \hbar} W_{\boldsymbol{\rho}_{1}}\left(p_{1}, q_{1}\right) W_{\boldsymbol{\rho}_{2}}\left(p_{1}, q_{1}\right) \\
& =\operatorname{Tr}\left(\boldsymbol{\rho}_{2} \boldsymbol{\rho}_{1}\right) .
\end{aligned}
$$

Os resultados aqui obtidos são de extrema valia no que se refere ao mapeamento das relações de comutação e anticomutação, conforme veremos a seguir. 


\section{O mapeamento das relações de comutação e anticomutação}

Para encontrarmos a transformada de Weyl das relações de comutação e anticomutação entre dois operadores arbitrários A e B em MQ, torna-se necessário estudar inicialmente a transformada de Weyl do produto AB. O ponto de partida é a representação integral (8), isto é,

$$
\begin{aligned}
\mathbf{A B} & =\int_{-\infty}^{\infty} \frac{d p_{1} d q_{1} d p_{2} d q_{2}}{(2 \pi \hbar)^{2}} \mathcal{A}\left(p_{1}, q_{1}\right) \mathcal{B}\left(p_{2}, q_{2}\right) \boldsymbol{\Delta}\left(p_{1}, q_{1}\right) \boldsymbol{\Delta}\left(p_{2}, q_{2}\right) \\
& =\int_{-\infty}^{\infty} \frac{d p d q}{2 \pi \hbar} f(p, q) \boldsymbol{\Delta}(p, q),
\end{aligned}
$$

no qual $f(p, q)=\operatorname{Tr}[\mathbf{A B} \boldsymbol{\Delta}(p, q)]$. Assim, substituindo a primeira igualdade da equação acima em $f(p, q)$, ficamos com

$$
\begin{aligned}
f(p, q) & =\int_{-\infty}^{\infty} \frac{d p_{1} d q_{1} d p_{2} d q_{2}}{(2 \pi \hbar)^{2}} \mathcal{A}\left(p_{1}, q_{1}\right) \mathcal{B}\left(p_{2}, q_{2}\right) \operatorname{Tr}\left[\boldsymbol{\Delta}\left(p_{1}, q_{1}\right) \boldsymbol{\Delta}\left(p_{2}, q_{2}\right) \boldsymbol{\Delta}(p, q)\right] \\
& =2^{2} \int_{-\infty}^{\infty} \frac{d p_{1} d q_{1} d p_{2} d q_{2}}{(2 \pi \hbar)^{2}} \mathcal{A}\left(p_{1}, q_{1}\right) \mathcal{B}\left(p_{2}, q_{2}\right) \exp \left\{\frac{2 i}{\hbar}\left[\left(q_{1}-q\right)\left(p_{2}-p\right)-\left(q_{2}-q\right)\left(p_{1}-p\right)\right]\right\}
\end{aligned}
$$

Para resolvermos essa integral devemos, em princípio, realizar a troca de variáveis $\bar{p}=p_{2}-p$ e $\bar{q}=q_{2}-q$, o que nos leva a

$$
f(p, q)=2^{2} \int_{-\infty}^{\infty} \frac{d p_{1} d q_{1} d \bar{p} d \bar{q}}{(2 \pi \hbar)^{2}} \mathcal{A}\left(p_{1}, q_{1}\right) \mathcal{B}(\bar{p}+p, \bar{q}+q) \exp \left\{\frac{2 i}{\hbar}\left[\bar{p}\left(q_{1}-q\right)-\bar{q}\left(p_{1}-p\right)\right]\right\} .
$$

Agora, expandindo a função $\mathcal{B}(\bar{p}+p, \bar{q}+q)$ em uma série de Taylor nas proximidades dos pontos $(p, q)$, obtem-se

$$
\mathcal{B}(\bar{p}+p, \bar{q}+q)=\sum_{n=0}^{\infty} \frac{1}{n !}\left(\bar{p} \frac{\partial}{\partial p}+\bar{q} \frac{\partial}{\partial q}\right)^{n} \mathcal{B}(p, q)=\exp \left(\bar{p} \frac{\partial}{\partial p}+\bar{q} \frac{\partial}{\partial q}\right) \mathcal{B}(p, q) .
$$

Esta relação permite-nos verificar a igualdade

$$
\begin{aligned}
& \exp \left\{\frac{2 i}{\hbar}\left[\bar{p}\left(q_{1}-q\right)-\bar{q}\left(p_{1}-p\right)\right]\right\} \exp \left(\bar{p} \frac{\partial}{\partial p}+\bar{q} \frac{\partial}{\partial q}\right) \mathcal{B}(p, q)= \\
& \exp \left\{\frac{2 i}{\hbar}\left[\bar{p}\left(q_{1}-q\right)-\bar{q}\left(p_{1}-p\right)\right]\right\} \exp \left[\frac{\hbar}{2 i}\left(\frac{\overleftarrow{\partial}}{\partial p} \frac{\vec{\partial}}{\partial q}-\frac{\overleftarrow{\partial}}{\partial q} \frac{\vec{\partial}}{\partial p}\right)\right] \mathcal{B}(p, q)
\end{aligned}
$$

que é fundamental para os nossos propósitos. Note que as variáveis $\bar{p}$ e $\bar{q}$ foram substituídas pelos operadores diferenciais $-\frac{\hbar}{2 i} \frac{\partial}{\partial q}$ e $\frac{\hbar}{2 i} \frac{\partial}{\partial p}$, respectivamente, atuando na primeira exponencial. Este fato justifica a taquigrafia utilizada no lado direito da igualdade e as setas indicam o sentido de atuação dos operadores diferenciais. Logo, a transformada de Weyl $f(p, q)$ pode ser reescrita como segue:

$$
\begin{aligned}
& f(p, q)= 2^{2} \int_{-\infty}^{\infty} \frac{d p_{1} d q_{1} d \bar{p} d \bar{q}}{(2 \pi \hbar)^{2}} \mathcal{A}\left(p_{1}, q_{1}\right) \exp \left\{\frac{2 i}{\hbar}\left[\bar{p}\left(q_{1}-q\right)-\bar{q}\left(p_{1}-p\right)\right]\right\} \\
& \times \exp \left[\frac{\hbar}{2 i}\left(\frac{\overleftarrow{\partial}}{\partial p} \frac{\vec{\partial}}{\partial q}-\frac{\grave{\partial}}{\partial q} \frac{\vec{\partial}}{\partial p}\right)\right] \mathcal{B}(p, q) \\
&= 2^{2} \int_{-\infty}^{\infty} \frac{d p_{1} d q_{1}}{2 \pi \hbar} \mathcal{A}\left(p_{1}, q_{1}\right) \underbrace{\int_{-\infty}^{\infty} \frac{d \bar{p} d \bar{q}}{2 \pi \hbar} \exp \left\{\frac{2 i}{\hbar}\left[\bar{p}\left(q_{1}-q\right)-\bar{q}\left(p_{1}-p\right)\right]\right\}}_{-\infty} \\
&= \int_{-\infty}^{\infty} d p_{1} d q_{1} \mathcal{A}\left(p_{1}, q\right) \\
&= \mathcal{A}(p, q) \exp \left[\frac{\hbar}{2 i}\left(\frac{\grave{\partial}}{\partial p} \frac{\vec{\partial}}{\partial q}-\frac{\grave{\partial}}{\partial q} \frac{\vec{\partial}}{\partial p}\right)\right] \mathcal{B}(p, q) \\
&\left.\left.\frac{\partial}{\partial p} \frac{\partial}{\partial q}-\frac{\grave{\partial}}{\partial q} \frac{\partial}{\partial p}\right)\right] \mathcal{B}(p, q) .
\end{aligned}
$$


Esse resultado nos leva a concluir que a transformada de Weyl do produto de operadores AB não é igual ao produto das transformadas $\mathcal{A}(p, q) \mathcal{B}(p, q)$, mas sim uma função mais complicada que depende de todas as ordens em $\hbar$ do operador pseudo-diferencial localizado entre as funções $\mathcal{A}(p, q)$ e $\mathcal{B}(p, q)$. Em geral, tal operador é denotado pelo símbolo * introduzido por Groenewold [23],

$$
\star \equiv \exp \left[\frac{\hbar}{2 i}\left(\frac{\overleftarrow{\partial}}{\partial p} \frac{\vec{\partial}}{\partial q}-\frac{\overleftarrow{\partial}}{\partial q} \frac{\vec{\partial}}{\partial p}\right)\right]
$$

o qual representa uma deformação pseudo-diferencial associativa do produto ordinário $\mathcal{A}(p, q) \mathcal{B}(p, q)$. Desse modo, a Eq.(40) também pode ser encontrada na literatura sob a forma [24]

$$
f(p, q)=\mathcal{A}(p, q) \star \mathcal{B}(p, q)
$$

cujas aplicações em física se estendem desde o estudo de modelos semiclássicos e caóticos até as idéias de geometria não-comutativa em gravitação quântica [25]. Outras aplicações do produto-^ e informações técnicas mais detalhadas podem ser obtidas nas referências [26, 27].

A transformada de Weyl das relações de comutação e anticomutação decorrem agora prontamente da Eq.(40),

$$
\begin{aligned}
{[\mathbf{A}, \mathbf{B}] } & \rightleftharpoons 2 i \sin \left[\frac{\hbar}{2}\left(\frac{\partial^{(\mathcal{A})}}{\partial p} \frac{\partial^{(\mathcal{B})}}{\partial q}-\frac{\partial^{(\mathcal{A})}}{\partial q} \frac{\partial^{(\mathcal{B})}}{\partial p}\right)\right] \mathcal{A}(p, q) \mathcal{B}(p, q), \\
\{\mathbf{A}, \mathbf{B}\} & \rightleftharpoons 2 \cos \left[\frac{\hbar}{2}\left(\frac{\partial^{(\mathcal{A})}}{\partial p} \frac{\partial^{(\mathcal{B})}}{\partial q}-\frac{\partial^{(\mathcal{A})}}{\partial q} \frac{\partial^{(\mathcal{B})}}{\partial p}\right)\right] \mathcal{A}(p, q) \mathcal{B}(p, q) .
\end{aligned}
$$

Nessas expressões, as funções seno e cosseno devem ser interpretadas formalmente como a série correspondente a cada um deles, respectivamente. Além disso, os índices $\mathcal{A}$ e $\mathcal{B}$ empregados nos operadores diferenciais indicam as transformadas de Weyl em que os operadores atuam, caracterizando assim numa mudança de notação. Alternativamente, utilizando a notação de Groenewold, tais expressões adquirem o seguinte aspecto:

$$
\begin{aligned}
{[\mathbf{A}, \mathbf{B}] } & \rightleftharpoons \mathcal{A}(p, q) \star \mathcal{B}(p, q)-\mathcal{B}(p, q) \star \mathcal{A}(p, q), \\
\{\mathbf{A}, \mathbf{B}\} & \rightleftharpoons \mathcal{A}(p, q) \star \mathcal{B}(p, q)+\mathcal{B}(p, q) \star \mathcal{A}(p, q) .
\end{aligned}
$$

Note que as Eqs.(42) e (43) são séries em $\hbar$ e os termos de ordem mais baixa representados por

$$
i \hbar\left(\frac{\partial^{(\mathcal{A})}}{\partial p} \frac{\partial^{(\mathcal{B})}}{\partial q}-\frac{\partial^{(\mathcal{A})}}{\partial q} \frac{\partial^{(\mathcal{B})}}{\partial p}\right) \mathcal{A}(p, q) \mathcal{B}(p, q) \quad \text { e } \quad 2 \mathcal{A}(p, q) \mathcal{B}(p, q)
$$

respectivamente. Consequentemente, a série associada a transformada de Weyl de $\frac{1}{i \hbar}[\mathbf{A}, \mathbf{B}]$ começa com o parênteses de Poisson das transformadas de Weyl de $\mathbf{A}$ e $\mathbf{B}$; enquanto que a série associada a $\frac{1}{2}\{\mathbf{A}, \mathbf{B}\}$, inicia com o produto das transformadas de Weyl de A e B. Assim, considerando o limite $\hbar \rightarrow 0$, ficamos com

$$
\begin{aligned}
& \lim _{\hbar \rightarrow 0} \frac{1}{i \hbar}[\mathbf{A}, \mathbf{B}] \longrightarrow\left(\frac{\partial^{(\mathcal{A})}}{\partial p} \frac{\partial^{(\mathcal{B})}}{\partial q}-\frac{\partial^{(\mathcal{A})}}{\partial q} \frac{\partial^{(\mathcal{B})}}{\partial p}\right) \mathcal{A}_{c l}(p, q) \mathcal{B}_{c l}(p, q) \\
& \lim _{\hbar \rightarrow 0} \frac{1}{2}\{\mathbf{A}, \mathbf{B}\} \longrightarrow \mathcal{A}_{c l}(p, q) \mathcal{B}_{c l}(p, q)
\end{aligned}
$$

Isto nos motiva a procurar o limite do análogo clássico da equação de von Neumann-Liouville, ou seja, verificar se a dinâmica quântica tende à dinâmica clássica no limite $\hbar \rightarrow 0$.

\section{A dinâmica quântica}

Vamos considerar um sistema físico qualquer descrito pelo operador densidade $\rho(t)=|\Psi(t)\rangle\langle\Psi(t)|$, em que $|\Psi(t)\rangle$ representa um estado puro do sistema. O cálculo do valor médio de um operador $\mathbf{F}$ é feito pela definição usual da MQ,

$$
\langle\mathbf{F}\rangle_{t}=\operatorname{Tr}[\boldsymbol{\rho}(t) \mathbf{F}]=\langle\Psi(t)|\mathbf{F}| \Psi(t)\rangle,
$$

ou através dos resultados obtidos até o presente momento,

$$
\langle\mathbf{F}\rangle_{t}=\int_{-\infty}^{\infty} \frac{d p d q}{2 \pi \hbar} f(p, q) W(p, q ; t)
$$


com $W(p, q ; t)$ sendo a função de Wigner para um tempo $t$ arbitrário. Assim, antes de prosseguirmos com a descrição da dinâmica do sistema, vamos apresentar algumas propriedades adicionais para a função de Wigner. As duas primeiras propriedades referem-se ao cálculo da quase-distribuição por intermédio das Eqs.(10) e (17), isto é,

$$
\text { (i) } \begin{aligned}
W(p, q ; t) & =\int_{-\infty}^{\infty} \frac{d v}{2 \pi \hbar} \exp \left(\frac{i}{\hbar} p v\right) \Psi(q-v / 2 ; t) \Psi^{*}(q+v / 2 ; t) \\
& =\int_{-\infty}^{\infty} \frac{d u}{2 \pi \hbar} \exp \left(\frac{i}{\hbar} q u\right) \Phi(p+u / 2 ; t) \Phi^{*}(p-u / 2 ; t)
\end{aligned}
$$

$$
\begin{aligned}
(\text { ii }) W(p, q ; t) & =2 \pi \hbar \exp \left(\frac{\hbar}{2 i} \frac{\partial^{2}}{\partial p \partial q}\right) \operatorname{Tr}[\boldsymbol{\rho}(t) \boldsymbol{\delta}(q-\mathbf{Q}) \boldsymbol{\delta}(p-\mathbf{P})] \\
& =\sqrt{2 \pi \hbar} \exp \left(\frac{\hbar}{2 i} \frac{\partial^{2}}{\partial p \partial q}\right)\left[\exp \left(\frac{i}{\hbar} p q\right) \Phi(p ; t) \Psi^{*}(q ; t)\right]
\end{aligned}
$$

Como o operador densidade é hermiteano, a função $W(p, q ; t)$ é real e consequentemente,

$$
W(p, q ; t)=\sqrt{2 \pi \hbar} \exp \left(-\frac{\hbar}{2 i} \frac{\partial^{2}}{\partial p \partial q}\right)\left[\exp \left(-\frac{i}{\hbar} p q\right) \Phi^{*}(p ; t) \Psi(q ; t)\right] .
$$

A terceira propriedade decorre da desigualdade de Cauchy-Schwarz, isto é,

$$
|W(p, q ; t)|^{2} \leq \int_{-\infty}^{\infty} \frac{d v}{2 \pi \hbar}|\Psi(q-v / 2 ; t)|^{2} \int_{-\infty}^{\infty} \frac{d u}{2 \pi \hbar}|\Psi(q+u / 2 ; t)|^{2}=\frac{1}{(\pi \hbar)^{2}}
$$

A desigualdade $|W(p, q ; t)| \leq \frac{2}{h}(h=2 \pi \hbar)$ implica que a função de Wigner é diferente de zero numa região cuja área do espaço de fase é menor ou igual a $\frac{h}{2}$ [21]. Portanto, tal função traz embutida a informação básica do princípio de incerteza: esta não pode ser localizada tanto em $p$ quanto em $q$, o que nos leva a concluir que as distribuições delta de Dirac estão descartadas neste contexto. De fato, este espaço de fase não é apenas uma outra representação do espaço de fase clássico, mas sim um representante legítimo de um espaço de estados com sua estrutura celulada devido ao princípio de incerteza [10]. Além disso, a função de Wigner $W(p, q ; t)$ pode assumir valores negativos, o que justifica a termos designado de quase-distribuição.

\section{IV.1 A equação de von Neumann-Liouville}

A evolução temporal da função de Wigner é uma consequência imediata da equação de von Neumann-Liouville para o operador densidade $\boldsymbol{\rho}(t)$,

$$
\frac{\partial}{\partial t} \boldsymbol{\rho}(t)=\frac{1}{i \hbar}[\mathbf{H}, \boldsymbol{\rho}(t)]
$$

no qual $\mathbf{H}$ é o hamiltoniano do sistema que independe do tempo. De fato, realizando-se a transformada de Weyl desta equação obtemos como resultado

$$
\frac{\partial}{\partial t} W(p, q ; t)=\frac{2}{\hbar} \sin \left[\frac{\hbar}{2}\left(\frac{\partial^{(H)}}{\partial q} \frac{\partial^{(W)}}{\partial p}-\frac{\partial^{(H)}}{\partial p} \frac{\partial^{(W)}}{\partial q}\right)\right] H(p, q) W(p, q ; t),
$$

sendo $H(p, q)$ a transformada de Weyl do operador H. Para resolver essa equação, vamos definir o operador Liouvilliano quântico

$$
\mathbf{L}(p, q)=\frac{2 i}{\hbar} \sin \left[\frac{\hbar}{2}\left(\frac{\partial^{(H)}}{\partial q} \frac{\partial^{(W)}}{\partial p}-\frac{\partial^{(H)}}{\partial p} \frac{\partial^{(W)}}{\partial q}\right)\right] H(p, q),
$$

o qual permite-nos reescrever a Eq.(55) da seguinte forma:

$$
\frac{\partial}{\partial t} W(p, q ; t)=-i \mathbf{L}(p, q) W(p, q ; t) .
$$

A solução formal desta equação é dada por

$$
W(p, q ; t)=\exp \left[-i \mathbf{L}(p, q)\left(t-t_{0}\right)\right] W\left(p, q ; t_{0}\right)
$$


Nesta expressão, observamos que a dependência em $\hbar$ da função de Wigner $W(p, q ; t)$ é proveniente de duas fontes: $W\left(p, q ; t_{0}\right)$ e $\mathbf{L}(p, q)$.

Para um sistema físico qualquer, cuja forma mapeada do operador hamiltoniano seja

$$
H(p, q)=\frac{p^{2}}{2 m}+V(q)
$$

a função de Wigner $W(p, q ; t)$ obedece a equação diferencial parcial [21]

$$
\left(\frac{\partial}{\partial t}+\frac{p}{m} \frac{\partial}{\partial q}-\frac{d V(q)}{d q} \frac{\partial}{\partial p}\right) W(p, q ; t)=\sum_{k=1}^{\infty} \frac{(-1)^{k}}{(2 k+1) !}\left(\frac{\hbar}{2}\right)^{2 k} \frac{d^{2 k+1} V(q)}{d q^{2 k+1}} \frac{\partial^{2 k+1}}{\partial p^{2 k+1}} W(p, q ; t),
$$

a qual é prontamente obtida através da Eq. (56). Ao fixarmos $\hbar=0$ nesta equação, o que é equivalente a considerarmos uma evolução clássica para $W(p, q ; t)$, verificamos que esta recupera a equação de Liouville da Mecânica Estatística Clássica,

$$
\left(\frac{\partial}{\partial t}+\frac{p}{m} \frac{\partial}{\partial q}-\frac{d V(q)}{d q} \frac{\partial}{\partial p}\right) W(p, q ; t)=0 .
$$

Portanto, o lado direito da Eq. (59) pode ser interpretado como um termo adicional que introduz correções quânticas à equação de Liouville Clássica.

Exemplo 4 A complexidade em resolver a equação diferencial parcial (59) está diretamente associada a natureza da energia potencial $V(q)$ do sistema físico que estamos abordando. Recentemente, alguns sistemas sujeitos a potenciais do tipo Morse, Pöschl-Teller e oscilador harmônico modificado (OHM), foram investigados e suas respectivas funções de Wigner calculadas através das funções de onda para os estados estacionários [28, 29]. Por exemplo, ao considerarmos

$$
V(q)=\left\{\begin{array}{lll}
D\left[\left(1-e^{-\beta q}\right)^{2}-1\right], & D>0 \text { e } \beta^{-1}>0 & \text { (Morse) } \\
-V_{0} \cosh ^{-2}(a q), & V_{0}=\hbar^{2} a^{2} / m \text { e } a>0 & \text { (Pöschl-Teller) } \\
\frac{\hbar^{2} \alpha^{2}}{2 m}\left[(\alpha q)^{2}-2(\alpha q) \tanh (\alpha q)\right], & \alpha>0 & \text { (OHM) }
\end{array}\right.
$$

temos que

$$
\frac{d^{2 k+1} V(q)}{d q^{2 k+1}}=\left\{\begin{array}{lll}
4 D \beta\left(2 \beta^{2}\right)^{k} e^{-3 \beta q / 2} \sinh \left(\frac{\beta q}{2}-k \ln 2\right), & \forall q & \text { (Morse) } \\
\frac{2 V_{0}}{a} \sum_{m=1}^{\infty}(-1)^{m}(2 a m)^{2(k+1)} e^{-2 a q m}, & q \geq 0 & \text { (Pöschl-Teller) } \\
-\frac{2 \hbar^{2} \alpha^{3}}{m} \sum_{m=1}^{\infty}(-1)^{m}(2 \alpha m)^{2 k}[1+2(k-\alpha q m)] e^{-2 \alpha q m}, & q>0 & \text { (OHM) }
\end{array}\right.
$$

para $k \geq 1$, o que nos leva a obter diferentes contribuições em ordens de $\hbar$ para cada potencial em questão. Em particular, as funções de Wigner para $t_{0}=0$ podem ser encontradas nas referências supracitadas, restando apenas realizar um estudo detalhado acerca da evolução temporal clássica e como as diferentes contribuições de $\hbar$ afetam tal evolução.

Em seguida, iremos investigar o limite $\hbar \rightarrow 0$ de $\mathbf{L}(p, q)$. Para tanto, vamos definir o operador

$$
\overleftrightarrow{\Lambda} \equiv \frac{\overleftarrow{\partial}}{\partial q} \frac{\vec{\partial}}{\partial p}-\frac{\overleftarrow{\partial}}{\partial p} \frac{\vec{\partial}}{\partial q}
$$

e introduzir um parâmetro adimensional $\alpha$ no Liouvilliano quântico de forma a reescrevê-lo como segue:

$$
\mathbf{L}_{\alpha}(p, q)=\frac{2 i}{\hbar \alpha} \sin \left(\frac{\hbar \alpha}{2} \overleftrightarrow{\Lambda}\right) H(p, q)
$$

Supondo que a função $H(p, q)$ não dependa de $\hbar$, a expansão em série de potências do operador

$$
\mathbf{L}_{\alpha}(p, q)=\frac{2 i}{\hbar \alpha}\left[\frac{\hbar \alpha}{2} \overleftrightarrow{\Lambda}-\frac{1}{3 !}\left(\frac{\hbar \alpha}{2}\right)^{3}(\overleftrightarrow{\Lambda})^{3}+\cdots\right] H(p, q)
$$

permite-nos realizar o limite $\alpha \rightarrow 0$ conforme o procedimento

$$
\lim _{\alpha \rightarrow 0} \mathbf{L}_{\alpha}(p, q)=i \overleftrightarrow{\Lambda} H(p, q)=\mathbf{L}_{0}(p, q)
$$


sendo $\mathbf{L}_{0}(p, q)$ um operador clássico. Nesse sentido, a evolução temporal (56) tem por solução

$$
W_{0}(p, q ; t)=\exp \left[-i \mathbf{L}_{0}(p, q)\left(t-t_{0}\right)\right] W\left(p, q ; t_{0}\right),
$$

ou considerando um passo anterior,

$$
\frac{\partial W_{0}}{\partial t}=\frac{\partial H}{\partial q} \frac{\partial W_{0}}{\partial p}-\frac{\partial H}{\partial p} \frac{\partial W_{0}}{\partial q}=\left\{H, W_{0}\right\}
$$

em que $\left\{H, W_{0}\right\}$ representa o parênteses de Poisson das funções $H(p, q)$ e $W_{0}(p, q ; t)$. Dessa forma, a Eq.(60) descreve a evolução clássica do objeto quântico $W\left(p, q ; t_{0}\right)$. Em suma, a introdução do parâmetro $\alpha$ nos leva a tratar de forma independente os limites $\hbar \rightarrow 0$ na função de Wigner e na sua evolução temporal [11].

Exemplo 5 Considere o operador hamiltoniano do oscilador harmônico unidimensional

$$
\mathbf{H}=\frac{\mathbf{P}^{2}}{2 m}+\frac{1}{2} m \omega^{2} \mathbf{Q}^{2},
$$

assim como a sua forma mapeada

$$
H(p, q)=\frac{p^{2}}{2 m}+\frac{1}{2} m \omega^{2} q^{2},
$$

a qual independe de $\hbar$. Para calcularmos a evolução temporal da função de Wigner $W_{n}(p, q ; t)$ através da Eq. (57), devemos em princípio estabelecer uma expressão formal para o operador Liouvilliano quântico $\mathbf{L}(p, q)$ associado ao sistema físico em estudo, que para o nosso caso é dado por

$$
\mathbf{L}(p, q)=i \omega\left[\frac{q}{b} \frac{\partial}{\partial(b p / \hbar)}-\frac{b p}{\hbar} \frac{\partial}{\partial(q / b)}\right]=\mathbf{L}_{0}(p, q) \quad\left(b^{2}=\hbar / m \omega\right) .
$$

Note que este operador não possui qualquer dependência em $\hbar$, levando-nos assim a uma evolução clássica da função de Wigner quântica $W_{n}(p, q)$ para $t_{0}=0$,

$$
W_{n}(p, q ; t)=\exp \left[-i t \mathbf{L}_{0}(p, q)\right] W_{n}(p, q),
$$

com $W_{n}(p, q)$ dado pela Eq. (31). Em seguida, vamos realizar a transformação de variáveis

$$
\left\{\begin{array} { l } 
{ \frac { q } { b } = \operatorname { R e } ( \alpha ) = r \operatorname { c o s } \theta } \\
{ \frac { b p } { \hbar } = \operatorname { I m } ( \alpha ) = r \operatorname { s i n } \theta }
\end{array} \quad \operatorname { c o m } \quad \left\{\begin{array}{l}
r^{2}=\frac{q^{2}}{b^{2}}+\frac{b^{2} p^{2}}{\hbar^{2}}=|\alpha|^{2} \\
\theta=\arctan \left(\frac{b^{2}}{\hbar} \frac{p}{q}\right)=\arctan \left[\frac{\operatorname{Im}(\alpha)}{\operatorname{Re}(\alpha)}\right]
\end{array}\right.\right.
$$

na Eq. (65), o qual permite-nos obter um resultado importante. Para tanto, devemos inicialmente recorrer as relações auxiliares

$$
\frac{\partial}{\partial(b p / \hbar)}=\sin \theta \frac{\partial}{\partial r}+\frac{\cos \theta}{r} \frac{\partial}{\partial \theta} \quad e \quad \frac{\partial}{\partial(q / b)}=\cos \theta \frac{\partial}{\partial r}-\frac{\sin \theta}{r} \frac{\partial}{\partial \theta},
$$

de maneira a reescrever o operador clássico $\mathbf{L}_{0}(p, q)$ como $\mathbf{L}_{0}(\theta)=i \omega \frac{\partial}{\partial \theta}$. Consequentemente, temos que

$$
W_{n}(r ; t)=\exp \left(-i \omega t \frac{\partial}{\partial \theta}\right) W_{n}(r)=W_{n}(r) \quad \Longrightarrow \quad W_{n}(p, q ; t)=W_{n}(p, q),
$$

ou seja, a função de Wigner associada ao autoestado do operador hamiltoniano (62) não evolui temporalmente. Este fato pode ser explicado com o auxílio do operador evolução temporal

$$
\mathbf{U}(t)=\exp \left(-\frac{i t}{\hbar} \mathbf{H}\right)=e^{-i \omega t / 2} \mathbf{R}(\omega t),
$$

no qual $\mathbf{R}(\omega t)=\exp \left(-i \omega t \mathbf{a}^{\dagger} \mathbf{a}\right)$ refere-se ao operador rotação, sendo

$$
\mathbf{a}=\frac{1}{\sqrt{2}}\left(\sqrt{\frac{m \omega}{\hbar}} \mathbf{Q}+i \frac{\mathbf{P}}{\sqrt{m \omega \hbar}}\right) \quad e \quad \mathbf{a}^{\dagger}=\frac{1}{\sqrt{2}}\left(\sqrt{\frac{m \omega}{\hbar}} \mathbf{Q}-i \frac{\mathbf{P}}{\sqrt{m \omega \hbar}}\right)
$$


os operadores aniquilação e criação, respectivamente. Dessa forma, ao calcularmos a função de Wigner $W_{n}(p, q ; t)$ por intermédio da Eq. (50),

$$
\begin{aligned}
W_{n}(p, q ; t) & =\int_{-\infty}^{\infty} \frac{d v}{2 \pi \hbar} \exp \left(\frac{i}{\hbar} p v\right)\langle q-v / 2|\mathbf{U}(t)| n\rangle\left\langle n\left|\mathbf{U}^{\dagger}(t)\right| q+v / 2\right\rangle \\
& =\int_{-\infty}^{\infty} \frac{d v}{2 \pi \hbar} \exp \left(\frac{i}{\hbar} p v\right)\left[e^{-i \omega t(n+1 / 2)}\langle q-v / 2 \mid n\rangle\right]\left[e^{i \omega t(n+1 / 2)}\langle n \mid q+v / 2\rangle\right] \\
& =\int_{-\infty}^{\infty} \frac{d v}{2 \pi \hbar} \exp \left(\frac{i}{\hbar} p v\right) \Psi_{n}(q-v / 2) \Psi_{n}^{*}(q+v / 2) \\
& =W_{n}(p, q)
\end{aligned}
$$

verificamos que o operador evolução temporal introduz apenas uma fase global e esta não apresenta nenhuma contribuição para o cálculo. De maneira análoga, o mesmo pode ser constatado para a Eq. (51),

$$
\begin{aligned}
W_{n}(p, q ; t) & =\sqrt{2 \pi \hbar} \exp \left(\frac{\hbar}{2 i} \frac{\partial^{2}}{\partial p \partial q}\right)\left[\exp \left(\frac{i}{\hbar} p q\right)\langle p|\mathbf{U}(t)| n\rangle\left\langle n\left|\mathbf{U}^{\dagger}(t)\right| q\right\rangle\right] \\
& =\sqrt{2 \pi \hbar} \exp \left(\frac{\hbar}{2 i} \frac{\partial^{2}}{\partial p \partial q}\right)\left\{\exp \left(\frac{i}{\hbar} p q\right)\left[e^{-i \omega t(n+1 / 2)}\langle p \mid n\rangle\right]\left[e^{i \omega t(n+1 / 2)}\langle n \mid q\rangle\right]\right\} \\
& =\sqrt{2 \pi \hbar} \exp \left(\frac{\hbar}{2 i} \frac{\partial^{2}}{\partial p \partial q}\right)\left[\exp \left(\frac{i}{\hbar} p q\right) \Phi_{n}(p) \Psi_{n}^{*}(q)\right] \\
& =W_{n}(p, q),
\end{aligned}
$$

corroborando assim com o resultado obtido anteriormente.

Agora, para encontrarmos uma expressão associada a derivada temporal do valor esperado de um observável F, basta lembrarmos da Eq.(49). Com isso, temos que

$$
\begin{aligned}
\frac{d}{d t}\langle\mathbf{F}\rangle_{t} & =\int_{-\infty}^{\infty} \frac{d p d q}{2 \pi \hbar} f(p, q) \frac{\partial}{\partial t} W(p, q ; t) \\
& =-i \int_{-\infty}^{\infty} \frac{d p d q}{2 \pi \hbar} f(p, q)[\mathbf{L}(p, q) W(p, q ; t)] \\
& =i \int_{-\infty}^{\infty} \frac{d p d q}{2 \pi \hbar}[\mathbf{L}(p, q) f(p, q)] W(p, q ; t) \\
& =-\frac{2}{\hbar} \int_{-\infty}^{\infty} \frac{d p d q}{2 \pi \hbar} \sin \left[\frac{\hbar}{2}\left(\frac{\partial^{(H)}}{\partial q} \frac{\partial^{(f)}}{\partial p}-\frac{\partial^{(H)}}{\partial p} \frac{\partial^{(f)}}{\partial q}\right)\right] H(p, q) f(p, q) W(p, q ; t)
\end{aligned}
$$

no qual a terceira igualdade foi obtida através da integração por partes e lembrando que $W(p, q ; t)$ se anula nos limites $p(q) \rightarrow \pm \infty$. Em particular, ao considerarmos os operadores momentum e posição, ficamos com

$$
\frac{d}{d t}\langle\mathbf{P}\rangle_{t}=-\int_{-\infty}^{\infty} \frac{d p d q}{2 \pi \hbar} \frac{\partial H}{\partial q} W(p, q ; t)
$$

e

$$
\frac{d}{d t}\langle\mathbf{Q}\rangle_{t}=\int_{-\infty}^{\infty} \frac{d p d q}{2 \pi \hbar} \frac{\partial H}{\partial p} W(p, q ; t)
$$

as quais representam a transformada de Weyl das equações de Ehrenfest da formulação usual da Mecânica Quântica. Para $\hbar \rightarrow 0$, a função de Wigner é equivalente ao produto $\delta\left(p-p_{0}\right) \delta\left(q-q_{0}\right)$ e as expressões acima recuperam as equações clássicas de Hamilton para $H_{c l}\left(p_{0}, q_{0}\right)$,

$$
\frac{d p_{0}}{d t}=-\frac{\partial H_{c l}}{\partial q_{0}} \quad \text { e } \quad \frac{d q_{0}}{d t}=\frac{\partial H_{c l}}{\partial p_{0}} .
$$

Resumindo, nesta última seção conseguimos recuperar a Mecânica Clássica e as equações fundamentais que governam a evolução temporal de valores médios dos observáveis físicos através de um processo limite formal, o qual permite obter uma descrição do espaço de fase clássico a partir de um espaço de fase quântico.

\section{Considerações Finais}

O objetivo central deste trabalho é apresentar uma contribuição para a literatura científica em língua portuguesa de um tema fascinante e de grande aplicabi- 
lidade nos diversos ramos da Física. Para tanto, utilizamos de alguns conceitos matemáticos que são geralmente ensinados nos cursos de Mecânica Quântica da graduação e pós-graduação, de modo a compilar um texto didático e rigoroso acerca da formulação de Weyl-Wigner da Mecânica Quântica no espaço de fase, possibilitando ao leitor uma rápida assimilação e um eventual aprofundamento no tema. De fato, para os leitores ávidos por informação recomendo a leitura das referências $[16,17]$ e mais recentemente, o excelente livro de W.P. Schleich [21] que traz um número razoável de aplicações em Óptica Quântica. Em suma, espero que o texto aqui apresentado cumpra sua finalidade e sirva de incentivo para alunos e professores na busca incessante por novos conhecimentos.

\section{Agradecimentos}

$\mathrm{O}$ autor gostaria de agradecer o convite feito pelo Prof. Esmerindo Bernardes (IFSC-USP) para ministrar um dos tópicos abordados no curso de verão e ao incentivo constante em escrever este material, ao Prof. Diógenes Galetti (IFT-UNESP) pela leitura cuidadosa e comentários pertinentes, além do apoio financeiro propiciado pela FAPESP através dos processos 01/11209-0 e $00 / 15084-5$.

\section{Referências}

[1] H. Weyl, Z. Phys. 46, 1 (1927).

[2] E. Wigner, Phys. Rev. 40, 749 (1932).

[3] A.M.O. de Almeida, Sistemas Hamiltonianos: Caos e Quantização, Editora da Unicamp, Campinas, segunda edição (1991).

[4] M.C. Gutzwiller, Chaos in Classical and Quantum Mechanics, Springer-Verlag, New York (1990).

[5] Y.S. Kim e M.E. Noz, Phase Space Picture of Quantum Mechanics (Group Theoretical Approach), Lecture Notes in Physics Series Vol. 40, World Scientific, Singapore (1991).

[6] D.T. Smithey, M. Beck, M.G. Raymer e A. Faridani, Phys. Rev. Lett. 70, 1244 (1993). Ver também: Ulf Leonhardt, Measuring the Quantum State of Light, Cambridge, New York (1997).

[7] D. Leibfried, D.M. Meekhof, B.E. King, C. Monroe, W.M. Itano e D.J. Wineland, Phys. Rev. Lett. 77, 4281 (1996).
[8] L.G. Lutterbach e L. Davidovich, Phys. Rev. Lett. 78, 2547 (1997)

[9] R.L. de Matos Filho e W. Vogel, Phys. Rev. A 58, R1661 (1998).

[10] D. Galetti, Mecânica Quântica no Espaço de Fase, Notas de aula da III Escola Mário Schenberg do Departamento de Física da UFPB, João Pessoa (1996).

[11] M.C. Tijero, Aproximações Semiclássicas nos Processos de Colisôes Atômico-Moleculares, Tese de doutoramento apresentada no Instituto de Física Teórica (UNESP), IFT-T.004/94 (1994).

[12] H. Weyl, The Theory of Groups and Quantum Mechanics, Dover, New York (1950).

[13] B. Leaf, J. Math. Phys. 9, 65 e 769 (1968).

[14] N.L. Balaz e B.K. Jennings, Phys. Rep. 104, 347 (1984).

[15] W.H. Louisell, Quantum Statistical Properties of Radiation, John Wiley \& Sons, New York (1990).

[16] M. Hillery, R.F. O'Connell, M.O. Scully e E.P. Wigner, Phys. Rep. 106, 121 (1984).

[17] H-W. Lee, Phys. Rep. 259, 147 (1995).

[18] I.S. Gradshteyn e I.M. Ryzhik, Table of Integrals, Series, and Products, Academic Press, San Diego (1980).

[19] M.O. Scully e M.S. Zubairy, Quantum Optics, Cambridge University Press, New York (1997).

[20] M. Orszag, Quantum Optics, Springer-Verlag, Berlin (2000).

[21] W.P. Schleich, Quantum Optics in Phase Space, WileyVCH, Berlin (2001).

[22] W. Vogel, D-G. Welsch e S. Wallentowitz, Quantum Optics: An Introduction, Wiley-VCH, Berlin (2001).

[23] H. Groenewold, Physica 12, 405 (1946).

[24] T. Curtright, T. Uematsu e C. Zachos, Generating all Wigner functions, hep-th/0011137 v2 (2001).

[25] L. Castellani, Class. Quant. Grav. 17, 3377 (2000).

[26] N.C. Dias e J.N. Prata, Causal Interpretation and Quantum Phase Space, quant-ph/0110062 (2001).

[27] O.V. Man'ko, V.I. Man'ko e G. Marmo, J. Phys. A: Math. Gen. 35, 699 (2002).

[28] A. Frank, A.L. Rivera e K.B. Wolf, Phys. Rev. A 61, 054102 (2000).

[29] G.W. Bund e M.C. Tijero, Phys. Rev. A 61, 052114 (2000). 